\title{
Wie generisch ist das generische Maskulinum? Über Genus und Sexus im Deutschen*
}

\author{
Ewa Trutkowski \\ Goethe-Universität Frankfurt am Main
}

This paper examines whether gender features (masculine, feminine, neuter) in German have to be interpreted semantically, along their specific gender, or whether they allow for a gender unrelated interpretation. As to this, two experiments with two different classes of nouns (gender marked and sex marked nouns vs. gender marked and sex neutral nouns) were conducted. The first experiment supports the view that in their function as nominal predicates masculine nouns, contrary to feminine (and neuter) nouns, have the widest extension - which confirms the existence of a 'Generic Masculine' (Generisches Maskulinum). On the other hand, the second experiment shows that in their function as subjects masculine nouns, contrary to feminine (and neuter) nouns, are the least flexible agreement controllers - hardly allowing for gender mismatches. Thus, masculine nouns behave differently depending on whether they appear as controllers/sources of agreement or as targets of agreement. The findings are supplemented by corpus data.

\section{Kurze Einführung in die Thematik}

Die Frage nach der Existenzberechtigung des generischen Maskulinums stellt sich nicht nur in linguistischen Fachkreisen, sondern prägt, wie das neuerliche Urteil des BGH (VI ZR 143/17, 13.03.2018) zeigt, auch eine gesellschaftliche Debatte.

Unter generische Maskulina fallen Nominalphrasen mit maskulinem Genus, welche auf die Gattung beziehungsweise typische Individuen referieren ohne Bezug auf den Sexus des/der dadurch Bezeichneten zu nehmen. Dementsprechend sind generische Maskulina ambige Ausdrücke, da sie sowohl auf männliche als auch auf nicht-männliche Individuen referieren können. Wie die Beispie-

Ich danke Markus Bader (Goethe-Universität Frankfurt am Main) für seine Unterstützung bei der statistischen Datenauswertung. 
le in (1a-d) illustrieren, können generische Maskulina im Singular oder im Plural auftreten, mit oder ohne Artikel:

(1) a. Heute hat sich nur ein Student beworben. ${ }^{1}$

b. Heute haben sich nur Studenten beworben.

c. Der Intellektuelle hat ausgedient.

d. Die Intellektuellen haben ausgedient.

Die oben erwähnte Ambiguität wird jedoch nicht von allen Mitgliedern der Sprechergemeinschaft geteilt: So geht das oben erwähnte Urteil auf eine Klägerin zurück, die sich durch generische Maskulina nicht angesprochen beziehungsweise nicht repräsentiert fühlt. Der BGH hingegen argumentiert, dass ,[d]er Bedeutungsgehalt grammatisch männlicher Personenbezeichnungen nach dem allgemein üblichen Sprachgebrauch und Sprachverständnis Personen umfassen [kann], deren natürliches Geschlecht nicht männlich ist ("generisches Maskulinum").“2

Wie die Beispiele (2)-(4) zeigen, können generische Maskulina (im Folgenden kursiv gesetzt) in unterschiedlichen syntaktischen Funktionen auftreten:

(2) Löwen sind wild. (Subjekt)

(3) Maria sucht einen Doktoranden. (Objekt)

(4) Hans und Maria sind Lehrer. (Prädikativum)

Im Folgenden sollen zwei Experimente vorgestellt werden, deren Ziel es war, die Interpretationsmöglichkeiten und das Kongruenzverhalten maskuliner (vs. femininer vs. neutraler) Nomen auszuloten. Als experimentelles Material wurden Prädikativkonstruktionen ähnlich wie (4) verwendet. Dies macht insofern Sinn, als das deutsche Verb nicht nach Genus kongruiert ${ }^{3}$ und Genus-Kongruenz nur über die Genusmerkmale des Prädikatsnomens abgelesen/determiniert werden kann. In beiden hier vorgestellten Experimenten wurde Kongruenz zwischen

1 Um die generische Lesart zu bekommen, muss die Betonung innerhalb der Nominalphrase ,ein Student' auf, Student' liegen.

2 Pressemitteilung des BGH Nr. 48/2018 vom 13.03.2018, http://juris. bundesgerichtshof.de/cgi-bin/rechtsprechung/document.py?Gericht=bgh\&Art=en\&Datum $=$ Aktuell\&Sort $=8193 \&$ Seite $=18 \& \mathrm{nr}=81591 \&$ linked $=$ pm\&Blank $=1$, link zuletzt überprüft am 28.03.2018.

3 Anders als zum Beispiel polnische Verben, welche in bestimmten Tempora (zum Beispiel im Präteritum) mit dem Subjekt nicht nur in Person und Numerus, sondern auch im Genus

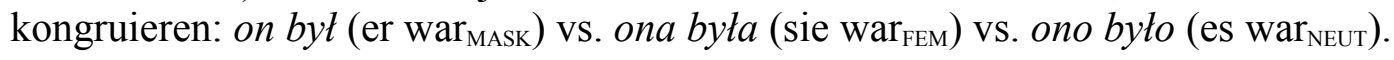


Subjekt und Prädikatsnomen untersucht, in Experiment 1 involvierte die Prädikativkonstruktion noch einen elliptischen Nachsatz.

\section{Experiment 1: Ellipsen}

Beobachtungen von Depiante \& Masullo (2001) für das Spanische und Merchant (2014:16) für das Griechische folgend kann ein maskuliner Antezedens eine Ellipse mit femininer Referenz lizenzieren, cf. (5), während ein femininer Antezedens eine Ellipse mit maskuliner Referenz nicht lizenzieren kann, cf. (6):

O Petros ine kalos dhaskalos, ala i Maria ine mia Der P. ist Lehrer $_{\mathrm{MASK}}$ Lehrer $_{\mathrm{MASK}}$ aber die M. ist eine $\mathrm{FEM}_{\mathrm{FM}}$ kakia.

schlechte $_{\mathrm{FEM}}$

'Petros ist ein guter Lehrer, aber Maria ist eine schlechte [Lehrerin].'

(6) *I Maria ine kali dhaskala, ala o Petros ine enas kakos. Die M. ist gut $_{\mathrm{FEM}}$ Lehrer $_{\mathrm{FEM}}$ aber der P. ist ein ${ }_{\mathrm{MASK}}$ schlechter $_{\mathrm{MASK}}$ 'Maria ist eine gute Lehrerin, aber Pedros ist ein schlechter [Lehrer].'

Solche Befunde sind mehr oder weniger erwartet, geht man davon aus, dass das maskuline Genus das weniger markierte ist (Sauerland 2008) und somit - im Gegensatz zum femininen - ohne eine spezifizierte Semantik daherkommt (siehe dazu Spathas \& Sudo 2015). Diese Feststellung lässt sich maßgeblich auf Jakobson (1971:221, auf einen Vortrag aus dem Jahr 1939 rekurrierend) zurückführen, der behauptet dass „das Maskulinum im Gegensatz zum Femininum ein Nullgenus [ist].“ Analoges wird auch für das Merkmal Person behauptet, zum Beispiel von Strukturalisten wie Benveniste (1971:198), der dafür plädiert, die 3. Person (im Gegensatz zur 1. und 2. Person) als Nicht-Person aufzufassen.

Der Nullgenus- beziehungsweise Nicht-Genus-Status des Maskulinums im Deutschen zeigt sich an vielerlei Phänomenen, man vergleiche zum Beispiel das obige Datum (4) mit seinem möglichen Pendant in (7): Hans und Maria können unter Lehrer, aber nicht unter Lehrerinnen subsumiert werden:

(4) Hans und Maria sind Lehrer.

(7) *Hans und Maria sind Lehrerinnen.

Für die Unmarkiertheit des Maskulinums gegenüber dem Femininum sprechen auch Daten wie (8)/(9): 
(8) Jeder strengt sich an, nur nicht Maria.

(9) *Jede strengt sich an, nur nicht Hans.

Und auch das unpersönliche Pronomen ,man' lässt sich als Marker einer genusmäßig nicht spezifizierten Semantik verstehen, cf. Als große Schwester hat man es nicht leicht.

Die Beobachtung, dass maskuline Referenten keine Prädikatsnomen mit femininem Genus dulden, cf. *Peter / Dieser Mann ist Akademische Rätin, feminine Referenten Prädikatsnomen mit maskulinem Genus jedoch schon, cf. Anna / Diese Frau ist Akademischer Rat mag streitbar sein. ${ }^{4}$ Merkmalstechnisch liegt in beiden Fällen ein Mismatch bezüglich der Genusmerkmale von Subjekt und Prädikativum vor. Da unter Ellipsen unterschiedliche Arten von Mismatches akkommodiert werden können, ${ }^{5}$ liegt es nahe, die Akzeptabilität solcher GenusMismatches auch in elliptischen Kontexten zu eruieren. Da die nachfolgend untersuchten elliptischen Konstruktionen analog zu den oben erwähnten von Merchant (2014) diskutierten Daten konstruiert sind, sollten die Akzeptabilitätsurteile hinsichtlich der deutschen Daten analog zu den griechischen (und spanischen) Daten ausfallen. Überdies wird erwartet, dass Muttersprachler des Deutschen das generische Maskulinum in Ellipsen (sowie darüber hinaus) verwenden und dementsprechend auch akzeptieren.

\subsection{Methode (Probanden, Material, Prozedur)}

An Experiment 1 nahmen 37 deutsche Muttersprachler teil, die Probanden wurden vor dem Experiment nicht über die damit verfolgten Ziele informiert.

Das Material für Experiment 1 bestand aus 16 Dialogen. Jeder Dialog lag in jeweils vier Versionen vor, die sich durch die vollständige Kombination der beiden Faktoren ,Genus Antezedens' (Maskulinum versus Femininum) und ,Genus Ellipse‘ (Maskulinum versus Femininum) ergaben. Das Prädikatsnomen bezeichnete zur Hälfte typisch männliche Berufe (wie zum Beispiel Pilot/-in) und zur Hälfte typisch weibliche Berufe (wie zum Beispiel Kosmetiker/-in). Jeder Dialog bestand aus zwei Sätzen. Der erste Satz bestand aus einem männlichen beziehungsweise weiblichen Eigennamen in Subjektfunktion, einem Au-

4 Für Mitglieder der Sprachgemeinschaft, welche das generische Maskulinum tolerieren (und verwenden), sollte der Satz Anna / Diese Frau ist Akademischer Rat akzeptabel sein.

5 So ist, wie Craenenbroeck \& Merchant (2013:710) zeigen, keine (morpho-)syntaktische Identität zwischen dem die Ellipse lizenzierendem Element (also dem Antezedens) und der Ellipse in (i) notwendig:

(i) Emily played beautifully at the recital and her sister will _, too.

(ii) Emily played beautifully at the recital and her sister will play beautifully at the recital, too. 
xiliar der 3. Person Indikativ Präsens und einem Prädikatsnomen mit maskulinen beziehungsweise femininen Genusmerkmalen. Der zweite Satz involvierte eine Ellipse des Prädikatsnomens und bestand aus einem männlichen beziehungsweise weiblichen Eigennamen in Subjektfunktion sowie dem Adverb auch . (10) und (11) zeigen zwei Originalsätze in allen vier Versionen.

(10) a. Anton ist Pilot. Peter auch.

b. Anton ist Pilot. Maria auch.

c. Anette ist Pilotin. Peter auch.

d. Anette ist Pilotin. Maria auch.

(11) a. Thomas ist Kosmetiker. Christoph auch.

b. Thomas ist Kosmetiker. Sabine auch.

c. Barbara ist Kosmetikerin. Christoph auch.

d. Barbara ist Kosmetikerin. Sabine auch.

Die Sätze wurden in Form eines Fragebogens präsentiert, der 82 Positionen enthielt (der Rest waren Ablenkersätze), wovon 16 Positionen Experiment 1 zugeordnet waren und von jeder Versuchsperson ${ }^{6}$ gesehen wurden. Jede der experimentellen Bedingungen kam gleich häufig vor. Insgesamt gab es zwölf verschiedene Fragebogenversionen.

Im Rahmen des Fragebogens wurden Akzeptabilitätsurteile abgefragt. Die Aufgabe an die Probanden ist in (12) wiedergegeben:

Ihre Aufgabe ist es, die Akzeptabilität von Sätzen auf einer Skala von 1 (völlig unakzeptabel) bis 7 (völlig akzeptabel) zu bewerten. Völlig akzeptabel ist ein Satz dann, wenn an ihm nichts auszusetzen ist. Völlig unakzeptabel ist ein Satz dagegen, wenn er so fehlerhaft ist, dass er in der Form niemals vorkommen würde.

Gehen Sie bei Ihrer Bewertung ausschließlich nach ihrem Gefühl vor und denken Sie nicht darüber nach, was man Ihnen in der Schule oder sonstwo über ,gutes“ oder ,schlechtes“ Deutsch erzählt hat. Es zählt ausschließlich Ihre persönliche Intuition!

6 Einzig Versuchsperson 29 sah nur zwölf Positionen, da sie den Fragebogen nicht vollständig ausgefüllt hatte. 


\subsection{Ergebnisse}

Die Durchschnittswerte für die jeweiligen Bedingungen sind der Tabelle $1 \mathrm{zu}$ entnehmen:

Tabelle 1: Durchschnittswerte für alle Bedingungen, Experiment 1

\begin{tabular}{lll}
\hline & \multicolumn{2}{l}{ GenusAntezedens } \\
\hline GenusEllipse & Mask & Fem \\
\hline Mask & 5.87 & 3.91 \\
\hline Fem & 4.55 & 5.88 \\
\hline
\end{tabular}

Die Daten wurden mittels einer zweifaktoriellen Varianzanalyse (ANOVA) analysiert.

In der Probanden-Analyse war der Faktor GenusAntezedens signifikant, in der Item-Analyse jedoch nur marginal signifikant $(\mathrm{F} 1(1,36)=10.609, \mathrm{p}<.05$; $\mathrm{F} 2(1,15)=3.112, \mathrm{p}=.098)$. Dabei wurden Sätze mit einem maskulinen Antezedens besser bewertet als solche mit einem femininen Antezedens (5.21 versus 4.99). Der Faktor GenusEllipse war in der Probanden-Analyse signifikant, in der Item-Analyse jedoch nicht $(\mathrm{F} 1(1,36)=9.810, \mathrm{p}<.05 ; \mathrm{F} 2(1,15)=1.517, \mathrm{p}=$ .237). Dabei wurden Sätze, bei denen die Ellipse einen femininen Referenten bezeichnete, besser bewertet als solche, bei denen die Ellipse einen maskulinen Referenten bezeichnete (5.21 versus 4.89). Die Interaktion zwischen den beiden Faktoren war sowohl in der Probanden-Analyse, als auch in der Item-Analyse signifikant $(\mathrm{F} 1(1,36)=24.683, \mathrm{p}<.001 ; \mathrm{F} 2(1,15)=820.207, \mathrm{p}<.001)$. Unter einem maskulinen Antezedens wurden Ellipsen, die auf eine maskuline Entität referierten, besser bewertet (5.87) als Ellipsen, die auf eine feminine Entität referierten (3.91). Unter einem femininen Antezedens wurden Ellipsen, die auf eine feminine Entität referierten, besser bewertet (5.88) als Ellipsen, die auf eine maskuline Entität referierten (4.55).

Im Einzelvergleich unterschieden sich alle Sätze mit Genus-Mismatches signifikant von den Sätzen ohne Genus-Mismatches (alle p-Werte $<0.001$ ), wobei die gemismatchten Sätze allesamt weniger akzeptabel bewertet wurden als die gematchten. Der interessante Einzelvergleich zwischen den beiden gemismatchten Sätzen, also der zwischen Sätzen der Art (10b) versus (10c) beziehungsweise (11b) versus (11c), war sowohl in der Probanden-Analyse signifikant $(\mathrm{t}=2.162, \mathrm{p}<.05)$, als auch in der Item-Analyse $(\mathrm{t}=2.437, \mathrm{p}<.05)$. Dabei wurden Ellipsen, die auf eine feminine Entität referierten und denen ein maskuliner Antezedens voranging, besser bewertet (4.55) als Ellipsen, die auf eine maskuline Entität referierten und denen ein femininer Antezedens voranging (3.91). 
Die Typisierungen nach typisch weiblichen versus typisch männlichen Berufen hatten keinen Einfluss auf die Ergebnisse.

\subsection{Diskussion}

Die Ergebnisse von Experiment 1 können wie folgt zusammengefasst und interpretiert werden: Am akzeptabelsten waren Mini-Dialoge, bei denen das Genus des Antezedens und der Ellipse identisch war. Fälle also, bei denen kein Mismatch vorlag. Strukturen, bei denen das Genus des Antezedens und der Ellipse nicht identisch war, wurden weniger akzeptabel bewertet. In Bezug auf diese gemismatchten Strukturen lässt sich zudem festhalten, dass Ellipsen mit femininer Referenz, die durch ein maskulines Antezedens lizenziert wurden, besser bewertet wurden als Ellipsen mit maskuliner Referenz, die durch ein feminines Antezedens lizenziert wurden. Dieses Ergebnis verifiziert die Hypothesen und Befunde verschiedener Autoren (zum Beispiel Jakobson 1971, Sauerland 2008, Merchant 2014, Spathas \& Sudo 2015), wonach das maskuline (im Vergleich zum femininen) Genus das weniger spezifizierte ist. Die Schlussfolgerung aus Experiment 1 lautet daher: Der von Merchant (2014) beobachtete Kontrast (siehe die Beispiele (5) versus (6)) liegt auch im Deutschen vor. Bezogen auf Prädikativkonstruktionen (die eine Ellipse des Prädikatsnomens involvieren) kann folgendes festgehalten werden:

(13) Auf feminine Nomen in Subjektfunktion kann mittels maskuliner Prädikativa referiert werden, auf maskuline Nomen in Subjektfunktion jedoch nicht mittels femininer Prädikativa.

Die Ergebnisse aus der Stichprobe sprechen für die Existenz eines generischen Maskulinums (und gegen ein generisches Femininum), zumindest was Daten mit Ellipsen in Prädikativkonstruktionen anbetrifft.

\section{Experiment 2: Sexus-neutrale Nomen}

Experiment 2 untersuchte das Kongruenzverhalten sexus-neutraler Appellativa, die (wie jedes singularische Nomen im Deutschen) über Genusmerkmale verfügen, deren Sexus jedoch nicht spezifiziert ist. Diese Nomen (zum Beispiel Waise, Gast oder Model) sind von sogenannten ,hybriden Appellativa ${ }^{7}$ wie Weib oder Mädchen zu unterscheiden. Bei Diewald (2017:19f) firmieren sexusneutrale Appellativa unter dem Begriff geschlechtsindifferente Substantive. Sie vermerkt, dass diese „völlig unabhängig vom grammatischen Genus - sowohl

7 Siehe dazu Nübling et al. (2013) mit zahlreichen Verweisen auf dazugehörige Literatur. 
auf männliche wie auch auf weibliche Lebewesen bezogen werden können. Die Bedeutung dieser Substantive macht über das lexikalische bzw. semantische Geschlecht schlicht keine Aussage.“ Ähnliches konstatieren auch Nübling et al. (2013:155) „Erst die Kenntnis der bezeichneten Person, des konkreten Referenten, offenbart den Sexus, nicht schon die Wortsemantik (wie bei Weib und Mädchen).“

Während bei hybriden Appellativa ein Konflikt zwischen grammatischem und semantischem Geschlecht vorliegt, können sexus-neutrale Nomen als Maskulinum oder Femininum (oder geschlechtsunspezifisch) interpretiert werden. Das „Problem“ bei sexus-neutralen Nomen ist also nicht die dem Genus zuwiderlaufende Interpretation, denn Lexeme der Art Gast, Person oder Model stellen per se Denotationen für Entitäten jedweder sexuellen Zuschreibung zur Verfügung. Vielmehr geht es um die Beantwortung der Frage, ob (die Absenz von) Sexus Einfluss auf die grammatische Kongruenz hat. Dabei sollten zwei relevante Dinge geklärt werden: Erstens, ob ein Genus-Mismatch zwischen Subjekt (als Controller of Agreement) und Prädikativum (als Target of Agreement) möglich ist, und zweitens, ob das spezifische Genus (Maskulinum, Femininum, Neutrum) sexus-neutraler Nomen dabei eine Rolle spielt.

\subsection{Methode (Probanden, Material, Prozedur)}

An Experiment 2 nahmen 30 deutsche Muttersprachler teil, die Probanden wurden vor dem Experiment nicht über die damit verfolgten Ziele informiert.

Das Material für Experiment 2 bestand aus 18 Sätzen. Jeder Satz bestand aus einem sexus-neutralen Nomen in Subjektfunktion, einem Auxiliar der 3. Person Indikativ Präsens und einem Substantiv in Prädikativfunktion, welches eine Nationalitätszugehörigkeit bezeichnete. Die Sätze wurden anhand zweier Faktoren variiert. Im Rahmen des Within-Faktors, Genus Prädikativ' wurde das Prädikatsnomen nach Genus variiert: eine Hälfte der Prädikatsnomen war maskulin (wie zum Beispiel Engländer), bei der anderen Hälfte der Prädikatsnomen kam noch das Movierungssuffix -in (wie in Engländerin) hinzu. Im Rahmen des Between-Faktors, Genus Subjekt ' wurde das Genus der sexus-neutralen Nomen variiert. Für jedes Genus von Nomen gab es sechs Sätze, sodass die sexusneutralen Nomen zu einem Drittel das Genus Femininum, zu einem Drittel das Genus Maskulinum und zu einem Drittel das Genus Neutrum trugen. Die Sätze in (14)-(16) sind Originalsätze aus dem Experiment.

(14) a. Diese Geisel FEM $_{1}$ ist Engländer ${ }_{\text {MASK. }}$.

b. Diese Geisel ${ }_{\mathrm{FEM}}$ ist Engländerin ${ }_{\mathrm{FEM}}$. 
(15) a. Dieser Gast MASK $_{\text {ist Waliser }}$ MASK.

b. Dieser Gast MASK $_{\text {ist Waliserin }}$ FEM .

(16) a. Dieses Opfer ${ }_{\mathrm{NEUT}}$ ist Österreicher ${ }_{\mathrm{MASK}}$.

b. Dieses Opfer $_{\mathrm{NEUT}}$ ist Österreicherin ${ }_{\mathrm{FEM}}$.

Die Sätze wurden in Form eines Fragebogens präsentiert, der 72 Positionen enthielt (der Rest waren Ablenkersätze), wovon 18 Positionen Experiment 2 zugeordnet waren und von jeder Versuchsperson gesehen wurden. Jede der experimentellen Bedingungen kam gleich häufig vor. Insgesamt gab es sechs verschiedene Fragebogenversionen. Es wurden wie in Experiment 1 Akzeptabilitätsurteile abgefragt. Die Aufgabe an die Probanden war identisch.

\subsection{Ergebnisse}

Die Durchschnittswerte für die jeweiligen Bedingungen sind der Tabelle $2 \mathrm{zu}$ entnehmen:

Tabelle 2: Durchschnittswerte für alle Bedingungen, Experiment 2

\begin{tabular}{lll}
\hline & \multicolumn{2}{l}{ GenusPrädikativ } \\
\hline GenusSubjekt & Mask & Fem \\
\hline Fem & 5.57 & 5.87 \\
\hline Mask & 5.72 & 4.00 \\
\hline Neut & 5.78 & 5.61 \\
\hline
\end{tabular}

Die Daten wurden mittels einer zweifaktoriellen Varianzanalyse (ANOVA) analysiert.

Die statistische Analyse ergab einen Haupteffekt des Faktors GenusPrädikativ $(\mathrm{F} 1(1,29)=12.55, \mathrm{p}<.01 ; \mathrm{F} 2(1,15)=7.048, \mathrm{p}<.05)$ sowie einen Haupteffekt des Faktors GenusSubjekt $(\mathrm{F} 1(1,58)=16.68, \mathrm{p}<.001 ; \mathrm{F} 2(1,15)=7.288$, $\mathrm{p}<.01)$. Die Interpretation der Haupteffekte ist an einer ebenfalls signifikanten Interaktion zwischen ihnen zu qualifizieren $(\mathrm{F} 1(1,58)=42.9, \mathrm{p}<.001 ; \mathrm{F} 2(1,15)$ $=9.390, \mathrm{p}<.01)$. Wie in Tabelle 2 zu sehen ist, wurden alle Sätze außer Sätze mit maskulinem Subjekt und femininem Prädikativum als sehr akzeptabel bewertet (5.57-5.87, alle p-Werte der Einzelvergleiche >0.1). Sätze mit maskulinem Subjekt und femininem Prädikativum erhielten dagegen nur eine Bewertung von 4.00, was signifikant schlechter ist als die Bewertungen in den anderen fünf Bedingungen (alle p-Werte der Einzelvergleiche $<0.05$ ). 


\subsection{Diskussion}

Den Befunden aus der Literatur zufolge ist das Maskulinum das am wenigsten spezifizierte und somit „flexibelste“ Genus, da maskuline Nomen in prädikativer Verwendung generisch interpretiert werden können (wie in Experiment 1 gezeigt), feminine Nomen jedoch nicht. Man würde daher erwarten, dass sich dieser Umstand in Experiment 2 bestätigt - was auch der Fall war: Analog zu Experiment 1 wurden Sätze mit maskulinem Prädikativ besser bewertet als solche mit femininen Prädikativ.

Doch so flexibel das Maskulinum in Prädikativfunktion (als Target of Agreement) auftritt, so rigide verhält es sich in Subjektfunktion (wo es als Controller/Source of Agreement fungiert): Das zeigt sich daran, dass Genus-Mismatches zwischen Subjekten mit maskulinem Genus und Prädikatsnomen mit nichtmaskulinem Genus am wenigsten akzeptabel bewertet wurden, cf. (17a). In Sätzen mit femininen (und neutralen) Subjekten hingegen, cf. (17b/c), wurden Genus-Mismatches mit einem Prädikatsnomen, das ein anderes Genus als das Subjekt trug, nicht sanktioniert:
a. * Dieser Gast $_{\mathrm{MASK}}$ ist Waliserin ${ }_{\mathrm{FEM}}$.
b. $\checkmark$ Diese Geisel $_{\mathrm{FEM}}$ ist Engländer MASK $_{\text {. }}$
c. $\checkmark$ Dieses $_{\text {Opfer }}$ NEUT ist Österreicher ${ }_{\mathrm{MASK}} /$ Österreicherin $_{\mathrm{FEM}}$

Aus den Ergebnissen dieses Experiments kann gefolgert werden, dass nur sexusneutrale maskuline Agreement Controller keine Genus-Mismatches mit einem Prädikatsnomen dulden. Agreement Controller mit nicht-maskulinem Genus hingegen erlauben Genus-Mismatches mit einem Prädikatsnomen. Die in (13) behauptete Schlussfolgerung aus Experiment 1 gilt somit (vorerst) auch für Experiment 2.

\section{Weitere Bemerkungen zu Experiment 1 und 2}

Oberflächlich betrachtet scheint es keine Unterschiede zwischen sexus-neutralen und Sexus realisierenden Nomen zu geben - vor allem, wenn man ausschließlich gemismatchte Strukturen betrachtet: (13) gilt für sexus-neutrale und nicht sexusneutrale Substantive.

(13) Auf feminine Nomen in Subjektfunktion kann mittels maskuliner Prädikativa referiert werden, auf maskuline Nomen in Subjektfunktion jedoch nicht mittels femininer Prädikativa. 
Nimmt man jedoch in Experiment 2 Sätze mit maskulinem Subjekt und femininem Prädikat (also Daten wie (17a)) aus der Masse aller Einzelvergleiche heraus, stellt man fest, dass sich Sätze mit Mismatches und Sätze ohne Mismatches nicht signifikant voneinander unterscheiden (alle p-Werte >0.1). Das heißt, femininen (und neutralen) Subjekten scheint es „egal“ zu sein, ob (beziehungsweise welche) Genus-Kongruenz mit dem Prädikatsnomen vorliegt, cf. die Daten in (14):
a. Diese Geisel $\mathrm{FEM}_{\mathrm{FM}}$ ist Engländer $\mathrm{MASK}_{\mathrm{K}}$.
b. Diese Geisel FEM $_{\text {ist Engländer }}$ MASK.

Dieser Befund aus Experiment 2 unterscheidet sich damit grundlegend von dem Analog-Befund aus Experiment 1: In Experiment 1 wurden die gemismatchten Dialoge mit femininen Subjekten und (elidierten) maskulinen Prädikatsnomen signifikant schlechter bewertet als die gematchten Dialoge mit femininen Subjekten und (elidierten) femininen Prädikatsnomen $(\mathrm{p}<.001)$.
a. Anton ist Pilot. Maria auch.
$(4.55)^{8}$
b. Anette ist Pilotin. Maria auch.

Der Vergleich zwischen den beiden Experimenten lässt den in (13) formulierten Schluss weiterhin zu. Darüber hinaus kann festgehalten werden, dass die Absenz von Sexus einen Einfluss auf die Genus-Kongruenz hat - Genus-Mismatches werden akzeptabler, wenn ein nicht-maskulines Subjekt keine Sexusmerkmale trägt (bei sexus-neutralen maskulinen Subjekten hingegen lässt sich kein Effekt beobachten).

Wendet man den Befund in (13) auf sexus-neutrale Nomen in Prädikativfunktion an, muss man jedoch feststellen, dass er Daten wie (19b) nicht erfassen kann:
a. Anna $\mathrm{FEM}_{\mathrm{Fe}}$ ist (ein netter) Gast $\mathrm{MASK}_{\text {. }}$
b. Peter ${ }_{\mathrm{MASK}}$ ist (ein netter) Gast $\mathrm{MASK}_{\mathrm{M}}$.

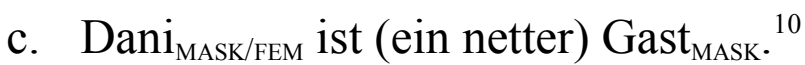

8 Ohne Ellipse liest sich (10b) wie folgt: Maria ${ }_{\mathrm{FEM}}$ ist auch Pilot ${ }_{\mathrm{MASK}}$.

9 Ohne Ellipse liest sich (10d) wie folgt: Maria ${ }_{\mathrm{FEM}}$ ist auch Pilotin ${ }_{\mathrm{FEM}}$.

10 Maskuline sexus-neutrale Nomen in Prädikativfunktion (wie zum Beispiel Gast, Star oder Säugling) verlangen eher nach einem in-/definiten Artikel beziehungsweise einem nachgestellten Attribut als ihre femininen und neutralen Pendants. Dafür habe ich aktuell keine Erklärung. 
(19) a. Anna $a_{\mathrm{FEM}}$ ist (eine kompetente) Fachkraft $\mathrm{FEM}_{\mathrm{FM}}$.

b. Peter $r_{\text {MASK }}$ ist (eine kompetente) Fachkraft FEM $_{\text {. }}$.

c. Dani MASK/FEM $_{\text {ist (eine kompetente) Fachkraft }}$ FEM .

(20) a. Anna FEM $_{\text {ist (ein tolles) Model }}$ NEUT.

b. Peter ${ }_{\mathrm{MASK}}$ ist (ein tolles) Model $_{\mathrm{NEUT}}$.

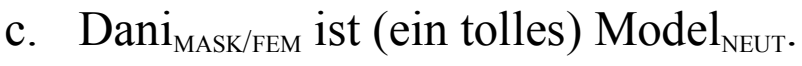

Vorkommen wie (19b) finden sich jedoch in großer Menge in Korpora ${ }^{11}$. Nachfolgend Beispiele für die Nomen Lehrkraft ${ }^{12}$ und Geisel ${ }^{13}$ :

(21) Damit wurde er anschließend Lehrkraft für "Bewegungskultur und gestaltung", also Tanzen. (Die Zeit, 20.03.2013, Nr. 11)

(22) »Mit ein bisschen Entgegenkommen ist er eine vollwertige Lehrkraft«, sagt sein Mentor. (Die Zeit, 05.01.2011, Nr. 02)

(23) Der Korrespondent des Pariser „Figaro“ war selbst Geisel radikaler Islamisten im Irak. (Der Tagesspiegel, 06.02.2005)

(24) Er ist eine Geisel in den Händen seiner vermeintlichen Freunde. (Berliner Zeitung, 24.01.2002)

Die obigen Korpusdaten und die Ergebnisse aus Experiment 1 legen nahe, dass sich sexus-neutrale Nomen wie Lehrkraft oder Geisel von nicht sexus-neutralen (movierten) Substantiven wie Pilotin oder Engländerin in Bezug auf Genuskongruenz unterscheiden, wenn sie als Prädikative maskuliner Subjekte fungieren, cf. (25):
a. Hans MASK $_{\text {ist (eine) Lehrkraft }}$ FEM.
b. ${ }^{*}$ Hans $_{\mathrm{MASK}}$ ist (eine) Lehrerin FEM $_{\text {. }}$.

Treten hingegen sexus-neutrale versus nicht sexus-neutrale Nomen mit maskulinem Genus (wie Gast versus Pilot) in Subjektfunktion auf, sind in Bezug auf

11 Für experimentelle Befunde zu Daten wie in (18)-(20) siehe Trutkowski (in Vorb.).

12 Korpustreffer für "@Lehrkraft", aus dem aggregierten Referenz- und Zeitungskorpus des Digitalen Wörterbuchs der deutschen Sprache, abgerufen am 28.03.2018.

13 Korpustreffer für "'"war \#<2 @Geisel"', aus dem aggregierten Referenz- und Zeitungskorpus des Digitalen Wörterbuchs der deutschen Sprache, abgerufen am 28.03.2018. 
Genuskongruenz mit dem Prädikativum keine Unterschiede festzustellen (siehe dazu auch die Ergebnisse der Experimente 1 und 2), cf. (26) und (27):
a. Hans $_{\mathrm{MASK}}$ ist Waliserin ${ }_{\mathrm{FEM}}$.
b. *Dieser Pilot $_{\mathrm{MASK}}$ ist Waliserin ${ }_{\mathrm{FEM}}$.
b. *Dieser Gast MASK $_{\text {ist Waliserin }}$ FEM.

(27) a. Hans $\mathrm{MASK}_{\text {ist (eine) Lehrkraft }}$ FEM / (ein) Model $_{\text {NEUT }}$.

b. Dieser Pilot MASK $_{\text {ist (eine) Lehrkraft }}$ FEM / (ein) Model $_{\text {NEUT }}$.

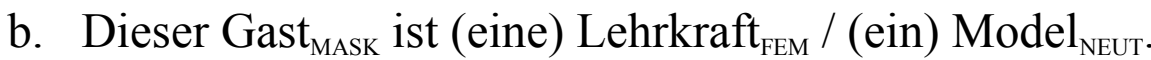

\section{$5 \quad$ Zusammenfassung}

Bezogen auf das Thema dieses Artikel bedeuten die hier vorgestellten Befunde zweierlei: Als Target of Agreement (in Prädikativfunktion) ist das Maskulinum das unterspezifizierteste / am wenigsten spezifizierte Genus: Kein anderes Genus verfügt über eine größere Extension. In diesem Sinn ist das Maskulinum im Gegensatz zum Femininum (und zum Neutrum) ein „hyperonymes“ Genus. Als Controller/Source of Agreement (in Subjektfunktion) stellt das Maskulinum das am meisten restringierte Genus dar: Kein anderes Genus kann so wenig gemismatcht werden, wie das Maskulinum. Grundsätzlich verlangen maskuline Subjekte maskuline Prädikative. Die einzige Option einer Abweichung von der ansonsten obligatorischen Genuskongruenz, die sich einem maskulinen Subjekt bietet, stellen sexus-neutrale Ausdrücke wie Lehrkraft oder abstrakte / vom deskriptiven Inhalt abstrahierende Ausdrücke (wie zum Beispiel Schimpfwörter, cf. Hans ist eine blöde Kuh), da das Merkmal Genus in diesen Fällen ohne Semantik (also ohne Sexus-Konnotation) daherkommt, was einen Mismatch ermöglicht und eine Abkehr von der in (13) formulierten Regel erlaubt.

\section{Literatur}

Benveniste, Émile. (1971). Problems in General Linguistics. Translated by Mary Elisabeth Meek. Cora Gables, FA. University of Miami Papers.

Craenenbroeck, Jeroen van \& Jason Merchant (2014). Ellipsis phenomena. In: Marcel den Dikken (ed.). The Cambridge Handbook of Generative Syntax. Cambridge: Cambridge University Press, 701-745.

Depiante, Marcela \& Pascal José Masullo (2001). Género y número en la elipsis nominal: consecuencias para la hipótesis lexicalista. In: Paper presented at the 1st Encuentro de Gramática Generativa. 
Diewald, Gabriele (2017). Richtig gendern. Wie Sie angemessen und verständlich schreiben. Berlin: Duden.

Jakobson, Roman (1971). Selected Writings II. Word and Language. The Hague. Paris: Mouton.

Merchant, Jason (2014). Gender mismatches under nominal ellipsis. In: Lingua 151 (2014) 932.

Nübling, Damaris, Simone Busley und Juliane Drenda (2013). Dat Anna und s Eva - Neutrale Frauenrufnamen in deutschen Dialekten und im Luxemburgischen zwischen pragmatischer und semantischer Genuszuweisung. In: Zeitschrift für Dialektologie und Linguistik 80/2, 152-196.

Sauerland, Uli (2008). On the semantic markedness of Phi-features. In: Daniel Harbour, David Adger \& Susana Bejar (eds.). Phi Theory. Phi-Features across Modules and Interfaces. Oxford: Oxford University Press, 57-82.

Spathas, Giorgos \& Yasutada Sudo (2015). Gendered Nouns and Nominal Ellipsis in Greek. https://semanticsarchive.net/Archive/zZiZDYxN/GenderedNounsGreek.pdf (zuletzt überprüft am 28.03.2018).

Trutkowski, Ewa (in Vorb.). On Gender and Sex in German. Manuskript, Goethe-Universität Frankfurt am Main. 